\title{
Induced pluripotent stem cells generated without viral integration
}

\author{
Matthias Stadtfeld ${ }^{1,2}$, Masaki Nagaya ${ }^{3}$, Jochen Utikal ${ }^{1,2}$, Gordon Weir $^{3}$, and Konrad \\ Hochedlinger ${ }^{1,2, \#}$ \\ ${ }^{1}$ Massachusetts General Hospital Cancer Center and Center for Regenerative Medicine 185 \\ Cambridge Street, Boston, MA 02114, USA \\ ${ }^{2}$ Harvard Stem Cell Institute \\ ${ }^{3}$ Section on Islet Transplantation and Cell Biology, Joslin Diabetes Center, One Joslin Place, \\ Boston, MA 02115 USA
}

\section{Abstract}

Pluripotent stem cells have been generated from mouse and human somatic cells by viral expression of the transcription factors Oct4, Sox2, Klf4, and c-Myc. A major limitation of this technology is the use of potentially harmful genome-integrating viruses. Here, we generate mouse induced pluripotent stem cells (iPS) from fibroblasts and liver cells by using non-integrating adenoviruses transiently expressing Oct4, Sox2, Klf4, and c-Myc. These adenoviral iPS (AdenoiPS) cells show DNA demethylation characteristic of reprogrammed cells, express endogenous pluripotency genes, form teratomas, and contribute to multiple tissues, including the germline, in chimeric mice. Our results show that insertional mutagenesis is not required for in vitro reprogramming. Adenoviral reprogramming may provide an improved method for generating and studying patient-specific stem cells and for comparing embryonic stem (ES) cells and iPS cells.

The introduction of defined transcription factors into mouse and human somatic cells has recently been shown to reprogram the developmental state of mature cells into that of pluripotent embryonic cells, generating so-called "induced pluripotent stem (iPS) cells" (1). iPS cells have been generated from multiple cell types by viral expression of Oct4 and Sox2, combined with either Klf4 and c-Myc (1-11) or LIN28 and Nanog (12). iPS cells are molecularly and functionally highly similar to ES cells, which makes in vitro reprogramming an attractive approach to produce patient-specific stem cells for studying and potentially treating degenerative disease. Indeed, reprogrammed skin cells have recently been shown to alleviate the symptoms of Parkinson's disease (13) and sickle cell anemia (14) in mouse models. However, a major limitation of this technology is the use of viruses that integrate into the genome and are associated with the risk of tumor formation due to the spontaneous reactivation of the viral transgenes (8). The low efficiency of reprogramming (0.01-0.1\% of input cells) also raised the possibility that insertional mutagenesis may be a prerequisite for in vitro reprogramming (15). For example, the retroviral tagging of explanted hematopoietic stem cells has been previously shown to select for clones in which

\footnotetext{
\#corresponding author: khochedlinger@helix.mgh.harvard.edu.
} 
the retroviral construct had inserted proximal to self-renewal genes thus causing their activation (16). Whereas the sequencing of a limited number of insertion sites in iPS cells did not reveal common targets (17), this possibility has not been unequivocally ruled out yet (15).

Therefore, we set out to generate iPS cells from mouse somatic cells using adenoviral vectors that allow for transient, high-level expression of exogenous genes without integrating into the host genome. Specifically, we cloned the cDNAs for Oct4, Sox2, c-Myc, and Klf4 into replication-incompetent adenoviral vectors under the control of the human cytomegalovirus immediate early (hCMV IE) promoter (Supplemental Figure 1). Initial attempts to reprogram mouse tail-tip fibroblasts (TTFs) into iPS cells with adenoviruses expressing the four reprogramming factors were unsuccessful, possibly owing to the rapid dilution of the virally encoded proteins from the cells. We have previously shown that viral Oct 4 expression can be substituted by a doxycycline-inducible $O c t 4$ allele driven by a reverse-tetracycline dependent transactivator (rtTA) present in the ROSA26 locus (Oct $4^{\mathrm{IND}}$ ) $(6,18)$, and it has been reported that liver cells require lower numbers of viral integrations than fibroblasts to be reprogrammed (17). Therefore, we infected approximately 500,000 adherent $O c t 4^{\mathrm{IND}}$ mouse fetal liver cells with adenoviruses expressing Sox2, Klf4, and cMyc at multiplicities of infection (MOI, number of viral particles per cell) of 20-50. This led to an infection efficiency of approximately $40-50 \%$ of cells with each factor and an estimated infection efficiency of 20-30\% of cells expressing all three adenoviral transgenes (Supplemental Figure 2A, E). After culture of infected fetal liver cells for 24 to 30 days in the presence of doxycycline, nine iPS-like colonies emerged that expressed the pluripotency markers Sox 2 and SSEA-1 and could be expanded into stable ES cell-like lines, similar to iPS cells produced with retro- or lentiviral vectors $(5,19$; Figure 1A). These adenoviral iPS (Adeno-iPS) cells continued to grow in the absence of doxycycline, indicating that transgenic Oct4 expression is no longer required.

These results prompted us to test if postnatal tail fibroblasts carrying the Oct4-inducible allele were equally amenable to reprogramming into Adeno-iPS cells. Fibroblasts required MOIs of 50-250 to achieve an infection efficiency of $\sim 30 \%$ for each vector, resulting in an estimated 10-20\% infection efficiency for triple-infected cells (Supplemental Figure 2B, E). Infection of more than 1,000,000 neonatal Oct $4{ }^{\mathrm{IND}}$ TTFs harboring an Oct4-GFP reporter with adenoviruses expressing myc, Klf4, and Sox 2 in the presence of doxycycline gave rise to a single $\mathrm{GFP}^{+}$colony, that grew into a stable, doxycycline-independent line.

In order to identify an adult cell type that might not require transgenic Oct4 expression, we chose hepatocytes, which are highly permissive for adenoviral infection $(19,20)$. Indeed, MOIs of 1-4 were sufficient to infect 70-80\% of these cells with individual vectors, with an estimated 50-60\% of cells expressing all four viral reprogramming factors (Supplemental Figure 2C, D, E). After incubation of approximately 500,000 adult hepatocytes isolated from mice carrying the Oct4-GFP and ROSA26-rtTA alleles but lacking the Oct4-inducible allele with adenoviruses expressing c-myc, Klf4, Oct4, and Sox2, we obtained three colonies, all of which could be expanded into stable ES-like cell lines expressing the pluripotency markers Oct-4 and SSEA1 (Supplemental Figure 3). PCR fingerprinting of Adeno-iPS cells confirmed their derivation from hepatocytes rather than from a contaminating ES cell line 
(Supplemental Figure 4). This demonstrates that iPS-like cells can be produced from adult cells by adenoviral vectors alone in the absence of the transgenic Oct 4 allele.

Next, we tested whether Adeno-iPS cells had re-established pluripotency at the molecular level by examining the activity of ES cell-specific markers. Expression analysis for the endogenous Oct4, Klf4, Sox2, c-Myc and Nanog genes by qPCR gave signals that were indistinguishable from those of ES cells, consistent with faithful molecular reprogramming (Figure 1B). In agreement with this, the Oct4 and Nanog promoters became demethylated in Adeno-iPS cells to a similar extent as seen in ES cells whereas they remained hypermethylated in cultured fibroblasts and hepatocytes, indicating that Adeno-iPS cells had undergone successful epigenetic reprogramming (Figure 1C and Supplemental Figure 5). In contrast to freshly infected fibroblasts, viral transcripts could not be detected in any of the Adeno-iPS cell lines tested, suggesting that the viral vectors had been diluted from the cells over time (Figure 1D).

Adenoviral vectors can integrate into the genome of host cells at extremely low frequencies (21). To exclude the possibility of permanent viral integration, PCR analysis of genomic DNA isolated from Adeno-iPS cell clones was performed with primers recognizing the different cDNA expression cassettes (see Supplemental Figure 1 for the position of the sequences recognized by the primers). Whereas adenoviral vector DNA, used as a positive control, readily produced PCR signals, we were unable to amplify these PCR products from genomic DNA from any of the Adeno-iPS cells (Figure 2B). Southern blot analysis using the cDNAs of the four viral vectors as probes confirmed the PCR results and yielded no evidence for the continuous presence of the adenoviral sequences in the Adeno-iPS cells whereas the single-copy Oct4 transgenic allele integrated into to CollA locus could be readily detected in iPS cell clones generated from Oct4 ${ }^{\mathrm{IND}}$ cells (Supplemental Figure 6). To rule out genomic integration of adenoviral sequences other than the cDNAs, we performed Southern blot analysis using the BamHI-digested full-length vector as a probe. Again, we did not detect exogenous viral sequences in the genomes of Adeno-iPS cells, whereas adenoviral sequences were detectable in HEK cells, consistent with a previous report (22) (Figure 2C). Moreover, the pBluescript (pBS)-derived portion of the adenoviral vector probe cross-hybridized with the Oct4 transgene, which also carries the pBS backbone, giving rise to a specific $\sim 3 \mathrm{~kb}$ signal in the iPS cell lines derived from fetal liver and tail-tip fibroblasts and thus serving as an internal positive control (Figure 2C). Together, these results strongly suggest that viral integration is not required for the generation of iPS cells. Although highly unlikely, we cannot rule out the possibility that small pieces of adenoviral DNA have inserted into the genome of Adeno-iPS cells but remained undetected due to the detection limits of Southern blot analysis.

In order to ascertain the developmental potential of Adeno-iPS cells, we injected the cells into the flanks of SCID mice. All cell lines tested produced teratomas after 3-4 weeks, which upon histological examination showed differentiation into representative cell types of the three germ layers including muscle, cartilage, and epithelial cells, thus demonstrating the pluripotency of Adeno-iPS cells (Figure 3A-C. Also see Table 1 and Supplemental Table 1 for a summary of all Adeno-iPS lines). In addition, Adeno-iPS cells generated apparently normal postnatal chimeras following injection into blastocysts. One chimera, obtained after 
blastocyst injection of Adeno-iPS cells labeled with a lentivirus expressing the redfluorescent protein tdTomato, was sacrificed at birth to examine the contribution of AdenoiPS cells to different tissues. As shown in Figure 3D-I, a high degree of chimerism was seen in multiple tissues including the lungs, brain and heart. Adeno-iPS cells gave rise to highdegree coat color chimeras (Figure 3J, K) and differentiated into functional germ cells as evidenced by the derivation of $\mathrm{GFP}^{+}$blastocysts after breeding of a male chimera generated with TTF-derived Adeno-iPS cells with a wildtype female (Figure 3L-O). Moreover, adult male chimeras derived from FL-9 and TTF-1 Adeno-iPS cells gave rise to viable germline offspring that showed the iPS cell-specific agouti coat color after mating with BDF1 female mice (1/20 or 5\% of FL- 9 offspring and $11 / 11$ or $100 \%$ of TTF- 1 offspring). Together, these results indicate that Adeno-iPS cells share the same developmental potential as iPS cells obtained with integrating viruses. Importantly, we have not observed tumor formation in any of the 12 coat color chimeras ranging between 4 and 13 weeks of age.

The efficiency of deriving iPS-like cells from fetal liver cells, TTFs, and hepatocytes (see Supplemental Figure 7 for an illustration of the derivation process from the different cell types) was extremely low, ranging from less than $0.0001 \%$ to $0.001 \%$ (Table 1 ). This frequency is lower than that obtained with integrating viruses $(\sim 0.01$ to $0.1 \%)$ and is probably due to the fact that many cells do not maintain viral expression long enough to trigger entry into a state sustained by endogenous pluripotency factors $(23,24)$. This conclusion is supported by qPCR analysis for adenoviral gene expression, which is gradually lost in dividing fibroblasts (Supplemental Figure 8). It should be informative to test whether the low efficiency of adenoviral reprogramming can be increased by the use of chemical compounds as has been reported for retroviral reprogramming (25-27).

DNA content analysis showed that about 23\% (3 out of 13) of the 13 Adeno-iPS lines were tetraploid, which is not seen in iPS cells produced with retro- or lentiviral vectors (Supplemental Figure 9 and Table 1). We speculate that adenoviral reprogramming either induces cell fusion or, alternatively, selects for rare tetraploid cells pre-existing in the starting cell populations. Indeed, it has been shown that the frequency of polyploid hepatocytes increases with age (28).

Our results demonstrate the generation of iPS cells without the use of integrating viruses by employing either a combination of adenoviruses and an inducible transgene or adenoviruses alone. Our work shows that insertional mutagenesis is not required for in vitro reprogramming, and it provides a platform for studying the biology of iPS cells lacking viral integrations. For example, it should now be possible to assess if iPS cells and ES cells are equivalent at the molecular and functional levels. This comparison has not been possible so far because viral transgenes are expressed at low levels in iPS cells and their progeny, which may affect their molecular signatures as well as their differentiation behavior and developmental potential. If human iPS cells can be generated without genome-integrating viruses, these cells may allow for the generation of safer patient-specific cells and thus could have important implications for cell therapy. Before translating these observations into a therapeutic setting, however, it will be important to assess if human iPS cells generated without viral integration are indeed as potent as human ES cells. 


\section{Materials and Methods}

\section{Adenoviral vectors and infection}

For the cloning of adenoviral vectors, EcoRI fragments containing the respective cDNAs for c-myc (constitutively active human T58A variant), Klf4, Oct4 and Sox 2 were integrated into the EcoRI site of pHIHG-Ad2. A fragment containing the cDNA sequence was isolated from the resulting plasmids by MfeI/PacI digestion and used for electroporation of BJ5183 cells together with linearized vector pAd-ClaI. Correct clones were identified by HindIII digestion. Viral constructs were transfected into 293A cells, and viral particles purified by two cycles of $\mathrm{CsCl}$ gradient and titered by optical absorbance (1). Fetal liver cells and fibroblasts that carried the $O c t 4^{I N D}$ allele were infected with adenoviruses expressing c-myc, $\mathrm{Klf} 4$ and Sox 2 at MOIs specified in the text for 1 hour at $37^{\circ} \mathrm{C}, 5 \% \mathrm{CO}_{2}$. This was followed by two washes with PBS and continued culture in the appropriate culture medium (see below) containing doxycycline at a concentration of $1 \mu \mathrm{g} / \mathrm{ml}$. The media was changed every 2-3 days and doxycycline was withdrawn at the times indicated in Supplemental Figure 8. Hepatocytes that did not carry the $O c t 4^{I N D}$ allele were infected with adenoviruses expressing all four reprogramming factors (c-myc, Klf4, Oct4 and Sox2) at MOIs between 1-4. After infection and washing, cells were cultured in media lacking doxycycline.

\section{Isolation of primary cells}

Adherent fetal liver cultures were established as previously described (2). Briefly, embryos were harvested at embryonic day E13.5 and livers dissected, washed with HBSS and incubated for $10-15$ minutes at $37^{\circ} \mathrm{C}$ in $0.2 \%$ collagenase IV (Invitrogen), $1 \mathrm{mM}$ EDTA and $1 \mathrm{mM} \mathrm{MgCl} 2$. Single-cell suspensions were prepared by repetitive pipetting and filtering through a $40 \mu \mathrm{m}$ cell strainer. Cells were resuspended in DMEM containing 10\% FBS, 10ng/ml EGF, $10 \mathrm{ng} / \mathrm{ml} \mathrm{bFGF}, 20 \mathrm{ng} / \mathrm{ml} \mathrm{TGFa}, 0.5 \mu \mathrm{g} / \mathrm{ml}$ insulin and $40 \mathrm{ng} / \mathrm{ml} \mathrm{HGF}$ and plated onto collagen I-treated culture plates (Becton Dickinson). Adult hepatocytes were isolated from 2-4 month-old mice by two-step collagenase perfusion protocol using Blendzyme 3 (Roche) (3). The perfused and isolated liver was freed of gall bladder and connective tissue and digested for 5-7 minutes. Digestion was stopped by addition of icecold DMEM containing FBS, followed by mechanically shearing of the liver capsule to release the dissociated cells. The resulting cell suspension was passed through a $100 \mu \mathrm{m}$ cell strainer and centrifuged at $600 \mathrm{rpm}$ for 1 minute. The pellet was considered as the hepatocyte preparation. Hepatocytes were cultured in DMEM containing $10 \% \mathrm{FBS}, 10^{-7}$ dexamethasone, $10 \mathrm{ng} / \mathrm{ml}$ EGF, $0.5 \mu \mathrm{g} / \mathrm{ml}$ insulin on collage I-treated plates. Fibroblast cultures from tail-tip biopsies of neonatal mice were established as previously described (4).

\section{Southern blot analysis}

$15 \mu \mathrm{g}$ (for cDNA Southern blot) or $30 \mu \mathrm{g}$ (for whole vector Southern blot) of genomic DNA was digested with BamHI, separated on a 1\% agarose gel and blotted onto HybondXL membrane (Amersham Biosciences). For cDNA blots, probes were prepared by digesting retroviral pMX vectors containing the four reprogramming cDNAs (5) with NcoI/BamHI (for c-myc) or EcoRI (for Klf4, Oct4 and Sox2). For the whole vector blot, pAd-Oct4 was digested with BamHI. Individual fragments $<10 \mathrm{~kb}$, with the exception of the fragment containing exclusively Oct4 cDNA sequences, were purified using the QIAGEN gel 
purification. Labeling with ${ }^{32} \mathrm{P}-\mathrm{a}-\mathrm{dCTP}$ was done using the Prime-It® II Random Labeling Kit (Stratagene) following the manufacturer's instructions.

\section{PCR analyses}

PCR reactions to test for adenoviral integrations were set up using $100 \mathrm{ng}$ of genomic DNA isolated from Adeno iPS clones or $1 \mathrm{pg}$ of the four different adenoviral vector plasmid DNAs (corresponding to the equivalent of 1 integration per genome sequence) using the primers described in Supplemental Table 2. The PCR reaction consisted of 30 cycles of $95^{\circ} \mathrm{C}$ for 30 seconds, $62^{\circ} \mathrm{C}$ for 45 seconds and $72^{\circ} \mathrm{C}$ for 30 seconds. The products were resolved on a $2 \%$ agarose gel. PCR analyses of the ROSA26 locus were set up with $100 \mathrm{ng}$ of genomic DNA using the primers rosa1 ( $5^{\prime}$-AAA GTC GCT CTG AGT TGT TAT-3), rosa2 ( $5^{\prime}$-GCG AAG AGT TTG TCC TCA ACC- $3^{\prime}$ ) and rosa3 (5' -GGA GCG GGA GAA ATG GAT ATG-3). The PCR reaction consisted of 30 cycles of $94^{\circ} \mathrm{C}$ for 30 seconds, $60^{\circ} \mathrm{C}$ for 30 seconds and $72^{\circ} \mathrm{C}$ for 60 seconds.

\section{Teratoma formation}

iPS cells were harvested by trypsinization, preplated onto untreated culture plates to remove feeders and differentiated cells and injected into the flanks of NOD/SCID mice, using $~ 5$ million cells per injection. Mice were sacrificed 3 weeks later, teratomas isolated and processed for histological analysis.

\section{Production of chimeric mice}

Female BDF1 mice were superovulated with PMS and hCG and mated to BDF1 stud males. Zygotes were isolated from females with a vaginal plug 24 hour after hCG injection. After 3 days of in vitro culture in KSOM media, blastocysts were identified, injected with iPS cells and transferred into pseudopregnant recipient females. In some cases, the iPS cells were labeled with a lentivirus constitutively expressing the red-fluorescent reporter gene tdTomato prior to blastocyst injections (6). Pups were delivered by Cesarean section at embryonic day E19.5 and nurtured by foster mothers. Newborn chimeras generated with tdTomato-labeled iPS cells were sacrificed, fixed overnight in 1.5\% paraformaldehyde (PFA), equilibrated in 30\% sucrose solution and frozen in OCT compound. $10 \mu \mathrm{m}$ sections were prepared on a cryostat and visualized on a Leica DMI400B inverted fluorescence microscope using RFP and brightfield channels. To determine germline contribution, chimeric mice were mated with hormone-primed BDF1 females. Blastocysts were flushed from the uterus and visualized under a fluorescence microscope using GFP and brightfield channels.

\section{Immunofluorescence analysis}

iPS cells were cultured on pretreated cover slips, fixed with 4\% PFA and permeabilized with $0.5 \%$ Triton X-100. Cells were then stained with primary antibodies against mOct4 (Santa Cruz, sc-8628), mSox 2 (Chemicon, AB5603), HA (Abcam, ab18181, for c-myc detection) and FLAG (Sigma, F1804, for Klf4 detection) followed by staining with the respective secondary antibodies conjugated to Alexa Fluor 488 or Alexa Fluor 546 (Invitrogen). Nuclei were counterstained with DAPI (Invitrogen). Cells were imaged using a Leica DMI4000B 
inverted fluorescence microscope equipped with a Leica DFC350FX camera. Images were processed and analyzed using Adobe Photoshop software.

\section{RNA isolation and real-time quantitative PCR analysis}

RNA was isolated from cells using the TriPure reagent (Roche) followed by RNA clean up with the RNeasy Minikit (Qiagen). cDNA was produced with the First Strand cDNA Synthesis Kit (Roche). Real-time quantitative PCR reactions were set up in triplicates with the Brilliant II SYBR Green QPCR Master Mix (Stratagene) and run on a Mx3000P QPCR System (Stratagene). Primer sequences are listed in Supplemental Table 3.

\section{Bisulfite sequencing}

Bisulfite treatment of DNA was performed with the EpiTect Bisulfite Kit (Qiagen) according to manufacturer's instructions. Oct4 and Nanog promoter regions were amplified as previously described (7). Amplified PCR products were purified by using gel filtration columns, cloned into the pCR4-TOPO vector (Invitrogen), and sequenced with M13 forward and reverse primers.

\section{Supplementary Material}

Refer to Web version on PubMed Central for supplementary material.

\section{Acknowledgments}

We thank H. Hock for critical comments on the manuscript. We are especially grateful to J. Lee of the Harvard Gene Therapy Initiative for help with the generation of adenoviral vectors. M.S. was supported by a Schering postdoctoral fellowship, J.U was supported by a Mildred Scheel postdoctoral fellowship. Support to K.H. was from the NIH Director's Innovator Award, the Harvard Stem Cell Institute, the Kimmel Foundation and the V Foundation.

\section{References}

1. Takahashi K, Yamanaka S. Cell. Aug 25.2006 126:663-76. [PubMed: 16904174]

2. Stadtfeld M, Brennand K, Hochedlinger K. Curr Biol. May 21.2008

3. Hanna J, et al. Cell. Apr 18.2008 133:250-64. [PubMed: 18423197]

4. Eminli S, Utikal J, Arnold K, Jaenisch R, Hochedlinger K. Stem Cells. 2008 in press.

5. Maherali N, et al. Cell Stem Cell. 2008 in press.

6. Maherali N, et al. Cell Stem Cell. 2007; 1:55-70. [PubMed: 18371336]

7. Lowry WE, et al. Proc Natl Acad Sci U S A. Feb 26.2008 105:2883-8. [PubMed: 18287077]

8. Okita K, Ichisaka T, Yamanaka S. Nature. Jul 19.2007 448:313-7. [PubMed: 17554338]

9. Takahashi K, et al. Cell. Nov 30.2007 131:861-72. [PubMed: 18035408]

10. Wernig M, et al. Nature. Jul 19.2007 448:318-24. [PubMed: 17554336]

11. Park IH, et al. Nature. Jan 10.2008 451:141-6. [PubMed: 18157115]

12. Yu J, et al. Science. Dec 21.2007 318:1917-20. [PubMed: 18029452]

13. Wernig M, et al. Proc Natl Acad Sci U S A. Apr 15.2008 105:5856-61. [PubMed: 18391196]

14. Hanna J, et al. Science. Dec 21.2007 318:1920-3. [PubMed: 18063756]

15. Hawley RG. Mol Ther. Aug.2008 16:1354-5. [PubMed: 18660799]

16. Kustikova O, et al. Science. May 20.2005 308:1171-4. [PubMed: 15905401]

17. Aoi T, et al. Science (express. 200810.1126/science. 1154884 
18. Hochedlinger K, Yamada Y, Beard C, Jaenisch R. Cell. May 6.2005 121:465-77. [PubMed: 15882627]

19. Li Q, Kay MA, Finegold M, Stratford-Perricaudet LD, Woo SL. Hum Gene Ther. Aug.1993 4:403-9. [PubMed: 8399487]

20. Yamada S, et al. Endocr J. Dec.2006 53:789-95. [PubMed: 16983179]

21. Harui A, Suzuki S, Kochanek S, Mitani K. J Virol. Jul.1999 73:6141-6. [PubMed: 10364373]

22. Louis N, Evelegh C, Graham FL. Virology. Jul 7.1997 233:423-9. [PubMed: 9217065]

23. Brambrink T, et al. Cell Stem Cell. 2008; 2:151-159. [PubMed: 18371436]

24. Stadtfeld M, Maherali N, Breault DT, Hochedlinger K. Cell Stem Cell. 10.1016/j.stem. 2008.02.0012008

25. Huangfu D, et al. Nat Biotechnol. Jun 22.2008

26. Mikkelsen TS, et al. Nature. May 28.2008

27. Shi Y, et al. Cell Stem Cell. Jun 5.2008 2:525-8. [PubMed: 18522845]

28. Gupta S. Semin Cancer Biol. Jun.2000 10:161-71. [PubMed: 10936066] 

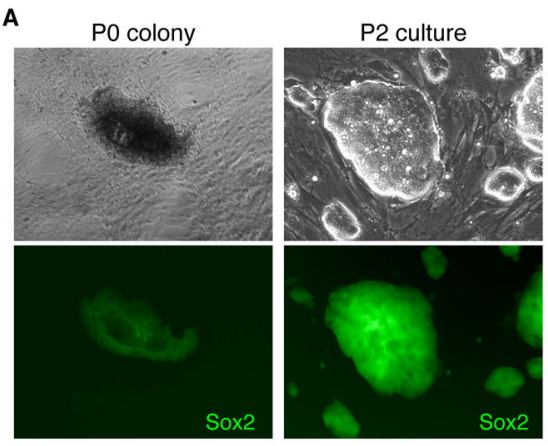

B

C
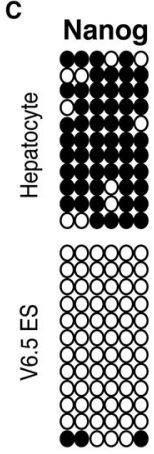

물

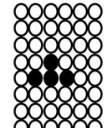

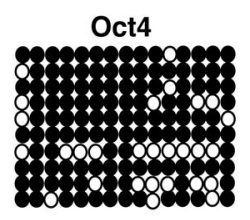

D
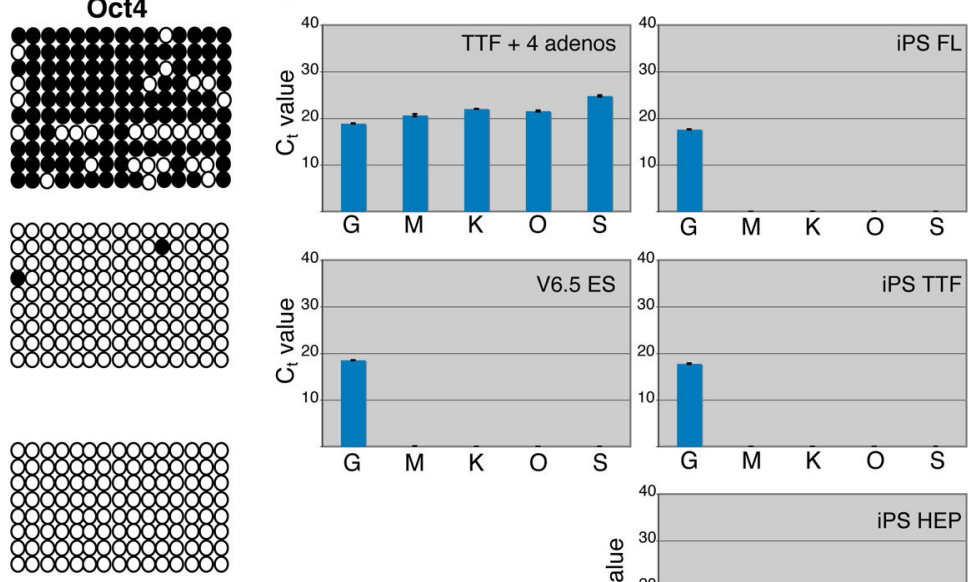

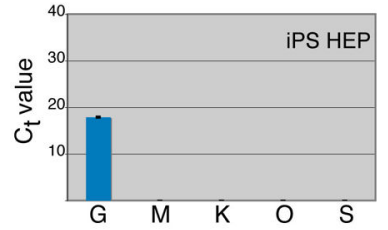

Figure 1. Analysis of pluripotency markers in Adeno-iPS cells

(A) Brightfield (upper panel) and fluorescence (lower panel) images of an Adeno-iPS cell clone established from Sox2-GFP fetal liver cells taken at passage 0 (P0) and passage 2 (P2). (B) Expression of endogenous $c-m y c, K l f 4$, Oct4, Sox2 and nanog measured by qPCR in Adeno-iPS cells derived from fetal liver (FL), fibroblasts (TTF) and hepatocytes (HEP) as well as in V6.5 control ES cells. (C) Bisulfite sequencing of the Oct4 and Nanog promoters in hepatocytes, ES cells and iPS cells derived from hepatocytes. Open circles represent unmethylated CpGs; closed circles denote methylated CpGs. (D) Expression levels of endogenous gapdh $(\mathrm{G})$ as well as adenoviral c-myc (M), Klf4 (K), Oct4 (O) and Sox2 (S) in fibroblasts three days after infection with adenoviruses (TTF +4 adenos), ES cells and Adeno-iPS cells derived from fetal liver, fibroblasts and hepatocytes. Error bars indicate one standard deviation. The absence of a bar indicates that the respective cDNA was not detected. 
A

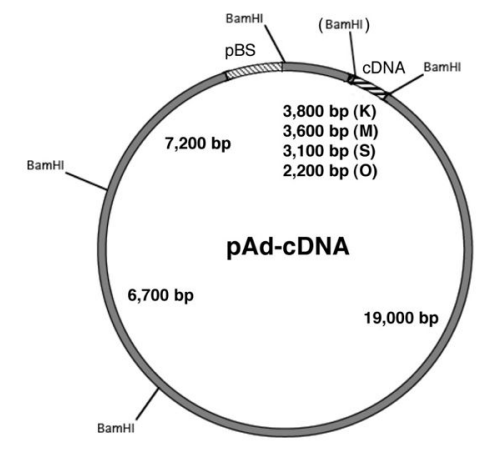

C

B

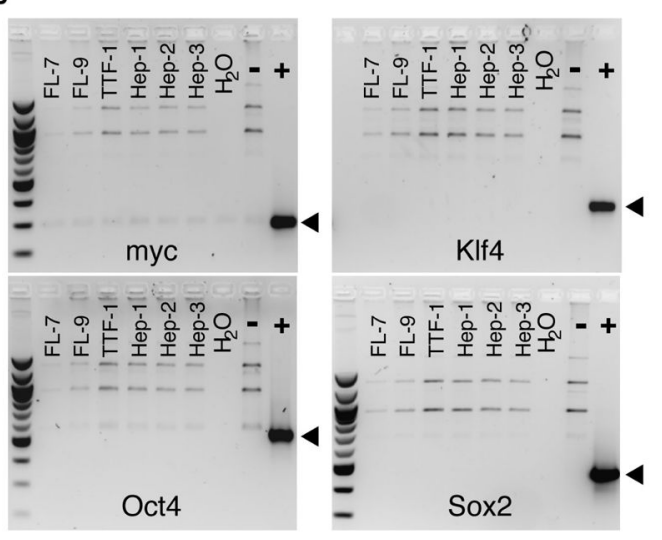

Figure 2. Absence of viral integration in Adeno-iPS cell

(A) Schematic drawing of the adenoviral vector indicating the position of the cDNA and the sizes of the respective DNA fragments after BamHI digestion. The bracketed BamHI site is only present in the Oct4 cDNA. A pBluescript (pBS) sequence present in both the adenoviral vector and the $O c t 4^{\mathrm{IND}}$ transgene is highlighted. (B) PCR analyses for adenoviral integration in genomic DNA from the indicated Adeno-iPS clones as well as from V6.5 ES cells that served as a negative control (-). Arrowhead indicates the position of the positive control band amplified from vector DNA (+). (C) Southern blot analysis of BamHI-digested genomic DNA using DNA fragments encompassing the entire adenoviral vector backbone isolated from pAd-Oct4 as probes. Plasmid DNA of pAd-Klf4 and pAd-Oct4 diluted to the equivalent of $0.2,1$ or 2.5 integrations per genome and genomic DNA of HEK cells (which contain adenoviral sequences in their genome) were used as positive controls. An asterisk (*) indicates bands resulting from hybridization of the pBS sequence in the adenoviral probe to transgenic sequences in the $O c t 4^{\mathrm{IND}}$ allele. Note that these bands are absent in HEP iPS, V6.5 ES and HEK cells. A double asterisk (**) indicates bands resulting from hybridization of parts of the probe to sequences present in the endogenous Oct4 locus or in Oct4 pseudogenes. These bands are present in all lanes containing genomic DNA including the wild type control and therefore serve as loading controls. Solid arrowheads indicate the position of BamHI fragments of the adenoviral vector and open arrowheads highlight adenoviral sequences detected in HEK cells. 

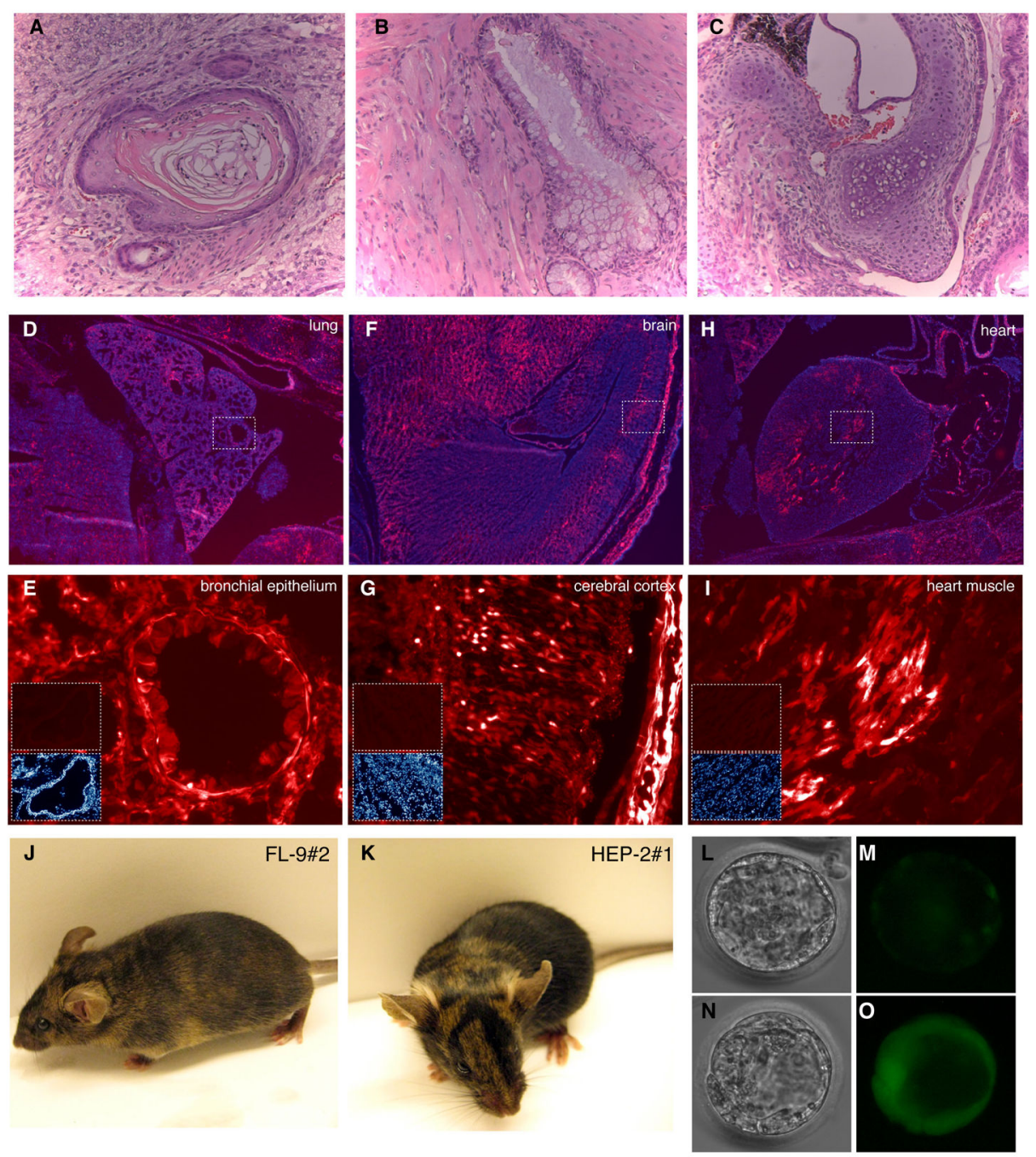

Figure 3. Pluripotency of Adeno-iPS cell

(A-C) Images of histological sections through teratomas formed by Adeno-iPS cells subjected to hematoxylin and eosin staining, showing keratinized epithelium (A), mucous epithelium (B) and cartilage (C). (D-I) Fluorescence images showing the contribution of red fluorescent protein-labeled Adeno-iPS cells to lung, brain and heart in a postnatal chimeric animal. Nuclei were counterstained with DAPI (blue). The small insets in (D, F, H) highlight the fields magnified in (E, G, I) whereas the insets in (E, G, I) show the background fluorescent levels and DAPI staining of corresponding tissues in a non-chimeric littermate. (J, K) Images of coat-color chimeras derived from fetal liver ( $\mathrm{J}$ ) and hepatocytes (K) Adeno-iPS cells. (L-O) Fluorescence and brightfield images of a wild type (L, M) blastocyst and an Oct4-GFP (N, O) blastocyst obtained after mating a chimeric mouse made with TTF-1 iPS cells expressing GFP from the Oct4 promoter with a wild type female. 


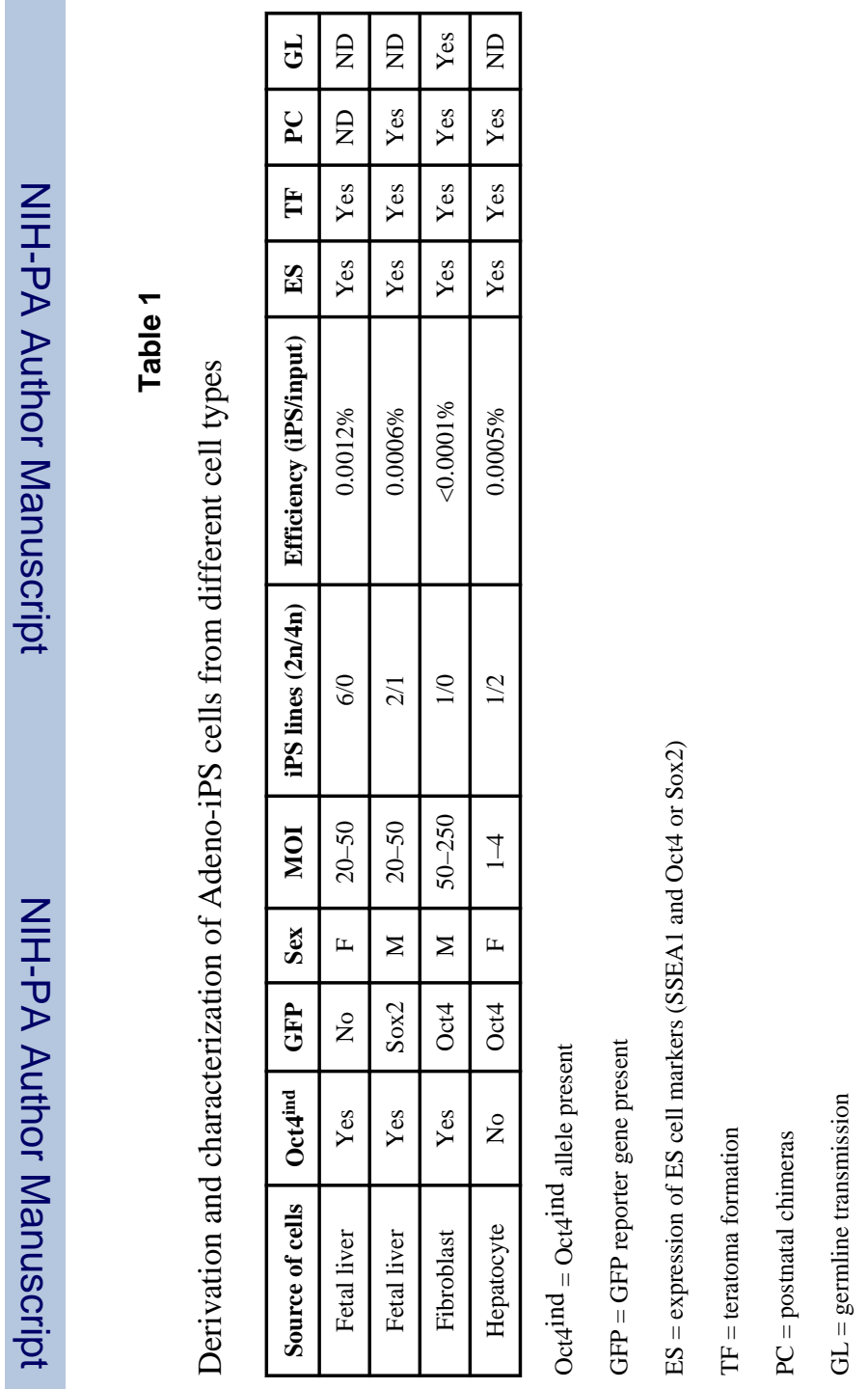

Science. Author manuscript; available in PMC 2014 April 15. 